\title{
Topology Between Two Sets
}

\author{
S. Nithyanantha Jothi ${ }^{1}$ and P. Thangavelu ${ }^{2 *}$ \\ 1 Department of Mathematics, Aditanar College, Tiruchendur - 628216, India \\ 2 Department of Mathematics, Karunya University, Coimbatore - 641 114, India \\ *Corresponding author: ptvelu12@gmail.com
}

Received: September 10, 2011 / Accepted: November 26, 2011

\begin{abstract}
The aim of this paper is to introduce a single structure which carries the subsets of $X$ as well as the subsets of $Y$ for studying the information about the ordered pair $(A, B)$ of subsets of $X$ and $Y$. Such a structure is called a binary structure from $X$ to $Y$. Mathematically a binary structure from $X$ to $Y$ is defined as a set of ordered pairs $(A, B)$ where $A \subseteq X$ and $B \subseteq Y$. The purpose of this paper is to introduce a new topology between two sets called a binary topology and investigate its basic properties where a binary topology from $X$ to $Y$ is a binary structure satisfying certain axioms that are analogous to the axioms of topology.
\end{abstract}

Keywords: Binary topology, binary open, binary closed, binary closure, binary interior, and binary continuity.

MSC 2010: 54A05, 54A99. 


\section{Introduction}

Point set topology deals with a nonempty set $X$ (Universal set) together with a collection $\tau$ of subsets of $X$ satisfying certain axioms. Such a collection $\tau$ is called a topological structure on $X$. General topologists studied the properties of subsets of $X$ by using the members of $\tau$. That is the information about a subset of $X$ can be known from the information of members of $\tau$. Therefore the study of point set topology can be thought of the study of information. But in the real world situations there may be two or more universal sets. If $A$ is a subset of $X$ and $B$ is subset of $Y$, the topological structures on $X$ and $Y$ provide little information about the ordered pair $(A, B)$. Our aim is to introduce a single structure which carries the subsets of $X$ as well as the subsets of $Y$ for studying the information about the ordered pair (A, B) of subsets of $X$ and $Y$. Such a structure is called a binary structure from $X$ to $Y$. Mathematically a binary structure from $X$ to $Y$ is defined as a set of ordered pairs $(A, B)$ where $A \subseteq X$ and $B \subseteq Y$. The concept of binary topology from $X$ to $Y$ is introduced and studied in section 2. The concepts of binary closed set, binary closure and binary interior are dealt in section 3 and the binary continuity is discussed in section 4 . For basic definitions and results of a topological space, the reader may refer Ryszard Engelking [1].

\section{Binary topology}

A binary topology from $X$ to $Y$ is a binary structure satisfying certain axioms that are analogous to the axioms of topology. In this section the concept of a binary topology between two non- empty sets is introduced and its structural properties are studied. Throughout this section $\wp(X)$ and $\wp(Y)$ denote the power sets of $X$ and $Y$ respectively.

Definition 2.1. Let $X$ and $Y$ be any two non empty sets. A binary topology from $X$ to $Y$ is a binary structure $M \subseteq \wp(X) \times \wp(Y)$ that satisfies the following axioms.

(i) $(\varnothing, \varnothing)$ and $(X, Y) \in M$.

(ii) $\left(A_{1} \cap A_{2}, B_{1} \cap B_{2}\right) \in M$ whenever $\left(A_{1}, B_{1}\right) \in M$ and $\left(A_{2}, B_{2}\right) \in M$.

(iii) If $\left\{\left(A_{\alpha}, B_{\alpha}\right): \alpha \in \Delta\right\}$ is a family of members of $M$, then

$$
\left(\bigcup_{\alpha \in \Delta} \mathrm{A}_{\alpha}, \bigcup_{\alpha \in \Delta} \mathrm{B}_{\alpha}\right) \in M \text {. }
$$

Definition 2.2. If $M$ is a binary topology from $X$ to $Y$ then the triplet $(X, Y, M)$ is called a binary topological space and the members of $M$ are called the binary open subsets of the 
binary topological space $(X, Y, M)$. The elements of $X \times Y$ are called the binary points of the binary topological space $(X, Y, M)$.

If $Y=X$ then $M$ is called a binary topology on $X$ in which case we write $(X, M)$ as a binary space.

Definition 2.3. Let $(X, Y, M)$ be a binary topological space and let $(x, y) \in X \times Y$. The binary open set $(A, B)$ is called a binary neighborhood of $(x, y)$ if $x \in A$ and $y \in B$.

Example 2.4. $I=\{(\varnothing, \varnothing),(X, Y)\}$ is called the indiscrete binary topology from $X$ to $Y$ and $(X, Y$, I) is called the indiscrete binary topological space.

Example 2.5. Let $D=\wp(X) \times \wp(Y)$. The binary topological space $(X, Y, D)$ is called the discrete binary topological space.

Example 2.6. Let $X=\{a, b, c, d\}$ and $Y=\{1,2,3,4,5\}$.

Let $M_{1}=\{(\varnothing, \varnothing),(\{a\},\{1\}),(\{b\},\{2\}),(\{a, b\},\{1,2\}),(X, Y)\}$ and

$M_{2}=\{(\varnothing, \varnothing),(\varnothing,\{1\}),(\{a\},\{1\}),(\{a\},\{1,2\}),(\{b\}, \varnothing),(\{b\},\{1\}),(\{b\},\{3\})$,

$$
\begin{gathered}
(\{b\},\{1,3\}),(\{a, b\},\{1\}),(\{a, b\},\{1,2\}),(\{a, b\},\{1,3\}), \\
(\{a, b\},\{(1,2,3\}),(X, Y)\} .
\end{gathered}
$$

Then $M_{1}$ and $M_{2}$ are binary topologies from $X$ to $Y$.

Example 2.7. Let $X=\{a, b, c\}$. Let $M=\{(\varnothing, \varnothing),(\{a\},\{c\}),(\{c\},\{b\}),(\{b\},\{a\}),(\{a, b\},\{a, c\})$, $(\{b, c\},\{a, b\}),(\{a, c\},\{b, c\}),(X, X)\}$. Then $M$ is a binary topology on $X$.

Example 2.8. Let $X, Y$ be any two non empty sets. Let $F$ be the set of all ordered pairs $(A, B)$ such that $A \subseteq X$ and $B \subseteq Y$ satisfying either $(A=B=\varnothing)$ or $(X \backslash A$ and $Y \backslash B$ are both finite) where $X \backslash A$ denotes the complement of $A$ in $X$. Then $F$ is a binary topology from $X$ to $Y$. This $F$ is called the co-finite binary topology from $X$ to $Y$.

Remark 2.9 If both $X$ and $Y$ are finite then the co-finite binary topology $F$ from $X$ to $Y$ is discrete one. 
Example 2.10. Let $X=\{0,1\} . S_{1}=\{(\varnothing, \varnothing),(\{0\},\{0\}),(X, X)\}, S_{2}=\{(\varnothing, \varnothing),(\{0\},\{1\}),(X, X)\}$, $S_{3}=\{(\varnothing, \varnothing),(\{1\},\{0\}),(X, X)\}$ and $S_{4}=\{(\varnothing, \varnothing),(\{1\},\{1\}),(X, X)\}$ are binary topologies on $X$, called Sierpinski binary topologies on $\{0,1\}$.

Example 2.11. Let $E_{1}$ denote the set of all real numbers. Let $S(x, r)=\left\{t \in E_{1}:|x-r|<r\right\}$ be an open ball for $x \in E_{1}$. Let $M_{1}=\left\{(A, B): A \subseteq E_{1}, B \subseteq E_{1}\right.$, and for every point $(x, y) \in(A, B)$ there are $r_{1}>0$ and $r_{2}>0$ with $\left.S\left(x, r_{1}\right) \subseteq A, S\left(y, r_{2}\right) \subseteq B\right\}$

Then $M_{1}$ is a binary topology on $E_{1}$ and it is called the Euclidean binary topology on $E_{1}$. The binary space $\left(E_{1}, M_{1}\right)$ is known as the Euclidean binary 1 -space. Analogously the Euclidean binary k-space namely $\left(E_{k}, M_{k}\right)$ can be defined.

Remark 2.12. As $\wp(X \times Y) \neq \wp(X) \times \wp(Y)$, the concept of binary topology from $X$ to $Y$ and the concept of topology on $X \times Y$ are independent. It is noteworthy to see that the product topology of topologies of $X$ and $Y$ is independent from the binary topology from $X$ to $Y$ as seen from the following example.

Suppose $(X, \tau)$ and $(Y, \sigma)$ are topological spaces. Let $\rho=\{A \times B: A \in \tau, B \in \sigma\}$. Then $\rho$ is the product topology for $X \times Y$. However this cannot be the binary topology from $X$ to $Y$ as $(\varnothing, \varnothing)$ does not belong to $\rho . \rho$ is a binary topology if we identify $(\varnothing, \varnothing)$ with the empty set $\varnothing$.

Now let $X=\{a, b, c\}$ and $Y=\{1,2,3,4\}$. Clearly $M=\{(\varnothing, \varnothing),(\{a\}, \varnothing)),(X, Y)\}$ is a binary topology from $X$ to $Y$. Since $(\{a\}, \varnothing))$ cannot be identified with $\{a\}$ or $\varnothing, M$ is not a product topology on $\mathrm{X} \times \mathrm{Y}$.

As the examples show, the sets $X$ and $Y$ may have many binary topologies. By regarding each binary topology from $X$ to $Y$ as a subset of $\wp(X) \times \wp(Y)$, the binary topologies from $X$ to $Y$ are partially ordered by set inclusion. If $M$ is any binary topology from $X$ to $Y$ then $I \subseteq M \subseteq D$. The following proposition can be easily established.

Proposition 2.13. Let $\left\{\Phi_{\alpha}: \alpha \in \Omega\right\}$ be any family of binary topologies from $X$ to $Y$. Then $\bigcap_{\alpha \in \Omega} \Phi_{\alpha}$ is also a binary topology from $X$ to $Y$ but $\bigcup \Phi_{\alpha}$ need not be a binary topology .

$$
\alpha \in \Omega
$$

Proof. Since each $\Phi_{\alpha}$ is a binary topology from $X$ to $Y$, we have $(\varnothing, \varnothing),(X, Y) \in \Phi_{\alpha}$ for all $\alpha \in \Omega$. This implies that $(\varnothing, \varnothing) \in \bigcap_{\alpha \in \Omega} \Phi_{\alpha}$ and $(X, Y) \in \bigcap_{\alpha \in \Omega} \Phi_{\alpha}$. 
Let $\left(A_{1}, B_{1}\right),\left(A_{2}, B_{2}\right) \in \bigcap_{\alpha \in \Omega} \Phi_{\alpha}$. Then $\left(A_{1}, B_{1}\right),\left(A_{2}, B_{2}\right) \in \Phi_{\alpha}$ for all $\alpha \in \Omega$. Since $\Phi_{\alpha}$ is a binary topology from $X$ to $Y$ for all $\alpha \in \Omega$, it follows that $\left(A_{1} \cap A_{2}, B_{1} \cap B_{2}\right) \in \Phi_{\alpha}$ for all $\alpha \in \Omega$ that implies $\left(A_{1} \cap A_{2}, B_{1} \cap B_{2}\right) \in \bigcap_{\alpha \in \Omega} \Phi_{\alpha}$. Let $\bigcap_{\alpha \in \Omega} \Phi_{\alpha}=\Phi$. Let $\left(A_{\beta}, B_{\beta}\right) \in \Phi$ for all $\beta \in \Delta$ where $\Delta$ is an arbitrary set. Then $\left(A_{\beta}, B_{\beta}\right) \in \Phi_{\alpha}$ for all $\alpha \in \Omega$ and for all $\beta \in \Delta$. Since each $\Phi_{\alpha}$ is a binary topology from $X$ to $Y$, it follows that $\left(\bigcup_{\beta \in \Delta} A_{\beta}, \bigcup_{\beta \in \Delta} B_{\beta}\right) \in \Phi_{\alpha}$ for all $\alpha \in \Omega$. Hence, $\left(\bigcup_{\beta \in \Delta} \mathrm{A}_{\beta}, \bigcup_{\beta \in \Delta} \mathrm{B}_{\beta}\right) \in \bigcap_{\alpha \in \Omega} \Phi_{\alpha}$. Thus $\bigcap_{\alpha \in \Omega} \Phi_{\alpha}$ is a binary topology from X to $\mathrm{Y}$. $\underset{\alpha \in \Omega}{\bigcup} \Phi \alpha$ is not a binary topology from $X$ to $Y$ as seen from Example 2.6.

Every binary topology from $X$ to $Y$ induce two topologies, one on $X$ and another on $Y$ as shown in the next proposition .

Proposition 2.14. Let $(X, Y, M)$ be a binary topological space. Then

(i) $\quad \tau(M)=\{A \subseteq X:(A, B) \in M$ for some $B \subseteq Y\}$ is a topology on $X$.

(ii) $\tau^{\prime}(M)=\{B \subseteq Y:(A, B) \in M$ for some $A \subseteq X\}$ is a topology on $Y$.

Proof. Clearly $\varnothing$ and $X \in \tau(M)$. Let $A_{1}, A_{2} \in \tau(M)$. Then $\left(A_{1}, B_{1}\right)$ and $\left(A_{2}, B_{2}\right) \in M$ for some subsets $B_{1}$ and $B_{2}$ of $Y$. Since $M$ is a binary topology, we have $\left(A_{1} \cap A_{2}, B_{1} \cap B_{2}\right) \in M$. Hence $A_{1} \cap A_{2} \in \tau(M)$. Let $\left\{A_{\alpha}: \alpha \in \Delta\right\}$ be a collection of elements of $\tau(M)$. Then for each $A_{\alpha} \subseteq X$ there is a $B_{\alpha} \subseteq Y$ with $\left(A_{\alpha}, B_{\alpha}\right) \in M$. Since $M$ is a binary topology, we get $\left(\bigcup_{\alpha \in \Delta} A_{\alpha}, \bigcup_{\alpha \in \Delta} B_{\alpha}\right) \in M$. This implies $\bigcup_{\alpha \in \Delta} \mathrm{A}_{\alpha} \in \tau(M)$ that implies that $\tau(M)$ is a topology on $X$. This proves (i).

Clearly $\varnothing$ and $Y \in \tau^{\prime}(M)$. Let $B_{1}, B_{2} \in \tau^{\prime}(M)$. Now, $B_{1} \in \tau^{\prime}(M)$ implies $B_{1} \subseteq Y$ and there exists $A_{1} \subseteq X$ such that $\left(A_{1}, B_{1}\right) \in M$. Also, $B_{2} \in \tau^{\prime}(M)$ implies $B_{2} \subseteq Y$ and there exists $A_{2} \subseteq X$ such that $\left(A_{2}, B_{2}\right) \in M$. Since $\left(A_{1} \cap A_{2}, B_{1} \cap B_{2}\right) \in M, B_{1} \cap B_{2} \in \tau^{\prime}(M)$. Now let $\left\{B_{\alpha}\right\}$ be a collection of elements of $\tau^{\prime}(M)$. Then for each $\alpha, B_{\alpha} \subseteq Y$ and $\left(A_{\alpha}, B_{\alpha}\right) \in M$ for some $A_{\alpha} \subseteq X$. Since $M$ is $a$ binary topology, we have $\left(\bigcup_{\alpha \in \Delta} \mathrm{A}_{\alpha}, \bigcup_{\alpha \in \Delta} \mathrm{B}_{\alpha}\right) \in M$ that implies $\bigcup_{\alpha \in \Delta} \mathrm{A}_{\alpha} \in \tau^{\prime}(M)$. This proves that $\tau^{\prime}(M$ ) is a topology on Y. 
The next proposition shows that a topology on $X$ and a topology on $Y$ induce a binary topology from $X$ to $Y$.

Proposition 2.15. Suppose $(X, \rho)$ and $(Y, \sigma)$ are two topological spaces. Then $\rho \times \sigma$ is a binary topology from $X$ to $Y$ such that $\tau(\rho \times \sigma)=\rho, \tau^{\prime}(\rho \times \sigma)=\sigma$.

Proof. Let $(X, \rho)$ and $(Y, \sigma)$ be two topological spaces.

Then $\rho \times \sigma=\{(A, B): A \in \rho, B \in \sigma\}$. We claim that $\rho \times \sigma$ is a binary topology from $X$ to $Y$. Clearly

$(\varnothing, \varnothing),(X, Y) \in \rho \times \sigma$. Let $\left(A_{1}, B_{1}\right)$ and $\left(A_{2}, B_{2}\right) \in \rho \times \sigma$.

Then $A_{1}, A_{2} \in \rho$ and $B_{1}, B_{2} \in \sigma$. Since $\rho, \sigma$ are topologies, we have $A_{1} \cap A_{2} \in \rho, B_{1} \cap B_{2} \in \sigma$. Therefore, $\left(A_{1} \cap A_{2}, B_{1} \cap B_{2}\right) \in \rho \times \sigma$.

Let $\left\{\left(A_{\alpha}, B_{\alpha}\right): \alpha \in \Delta\right\}$ be a family of members of $\rho \times \sigma$. Since $\rho, \sigma$ are topologies, we have $\bigcup_{\alpha \in \Delta} \mathrm{A}_{\alpha} \in \rho$ and $\bigcup_{\alpha \in \Delta} \mathrm{B}_{\alpha} \in \sigma$ that implies $\left(\bigcup_{\alpha \in \Delta} \mathrm{A}_{\alpha}, \bigcup_{\alpha \in \Delta} \mathrm{B}_{\alpha}\right) \in \rho \times \sigma$. This proves that $\rho \times \sigma$ is a binary topology from $X$ to $Y$. Next we show that $\tau(\rho \times \sigma)=\rho$ and $\tau^{\prime}(\rho \times \sigma)=\sigma$.

Now, $\tau(\rho \times \sigma)=\{A \subseteq X:(A, B) \in \rho \times \sigma \quad$ for some $B \subseteq Y\}$.

$A \in \rho \Rightarrow(A, B) \in \rho \times \sigma$ for every $B \in \sigma$ that $\Rightarrow A \in \tau(\rho \times \sigma)$. Therefore , $\rho \subseteq \tau(\rho \times \sigma)$.

Now, $A \in \tau(\rho \times \sigma) \Rightarrow\left(A, B_{1}\right) \in \rho \times \sigma$ for some $B_{1} \subseteq Y$ that $\Rightarrow B_{1} \in \sigma \Rightarrow A \in \rho$.

The above arguments show that $\tau(\rho \times \sigma)=\rho$.

$B \in \sigma \Rightarrow(A, B) \in \rho \times \sigma$ for every $A \in \rho$ that $\Rightarrow B \in \tau^{\prime}(\rho \times \sigma)$. Therefore, $\sigma \subseteq \tau^{\prime}(\rho \times \sigma)$.

Now, $B \in \tau^{\prime}(\rho \times \sigma) \Rightarrow\left(A_{1}, B\right) \in \rho \times \sigma$ for some $A_{1} \in \rho, A_{1} \subseteq X$ that implies $B \in \sigma$. Therefore,

$\tau^{\prime}(\rho \times \sigma) \subseteq \sigma$. This proves that $\tau^{\prime}(\rho \times \sigma)=\sigma$

\section{3 . Binary closed, binary closure and binary interior}

The binary complement of an element of $\wp(X) \times \wp(Y)$, is defined component wise. That is the binary complement of $(A, B)$ is $(X \backslash A, Y \backslash B)$. In this section the concepts of binary closed, binary closure and binary interior are introduced and their properties are discussed.

Definition 3.1. Let $(X, Y, M)$ be a binary topological space and $A \subseteq X, B \subseteq Y$. Then $(A, B)$ is binary closed in $(X, Y, M)$ if $(X \backslash A, Y \backslash B) \in M$. 
The proof for the next proposition is straight forward.

Proposition 3.2. Let $(X, Y, M)$ be a binary topological space. Then

(i) $(X, Y)$ and $(\varnothing, \varnothing)$ are binary closed sets.

(ii) if $\left(A_{1}, B_{1}\right)$ and $\left(A_{2}, B_{2}\right)$ are binary closed then $\left(A_{1} \cup A_{2}, B_{1} \cup B_{2}\right)$ is binary closed.

(iii) If $\left\{\left(\mathrm{A}_{\alpha}, \mathrm{B}_{\alpha}\right): \alpha \in \Delta\right\}$ is a family of binary closed sets, then $\left(\bigcap_{\alpha \in \Delta} \mathrm{A}_{\alpha}, \bigcap_{\alpha \in \Delta} \mathrm{B}_{\alpha}\right)$ is binary closed.

Definition 3.3. Let $(A, B)$ and $(C, D) \in \wp(X) \times \wp(Y)$. We say that $(A, B) \subseteq(C, D)$ if $A \subseteq C$ and $B$ $\subseteq \mathrm{D}$.

Proposition 3.4. Let $(X, Y, M)$ be a binary topological space and $(A, B) \subseteq(X, Y)$. Let $(A$,

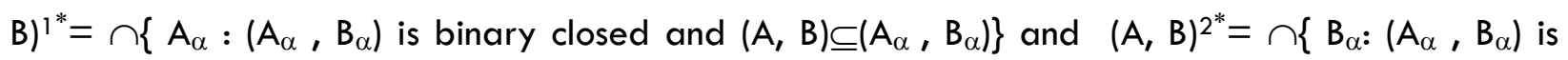
binary closed and $\left.(A, B) \subseteq\left(A_{\alpha}, B_{\alpha}\right)\right\}$. Then $\left((A, B)^{1^{*}},(A, B)^{2^{*}}\right)$ is binary closed and $(A, B) \subseteq((A$, $\left.B)^{1^{*}},(A, B)^{2^{*}}\right)$.

Proof. From Proposition 3.2 (iii), it follows that $\left((A, B)^{1^{*}},(A, B)^{2^{*}}\right)$ is binary closed and $(A, B) \subseteq$ $\left((A, B)^{1^{*}},(A, B)^{2^{*}}\right)$.

The above proposition motivates us to define the following concept.

Definition 3.5. The ordered pair $\left((A, B)^{1^{*}},(A, B)^{2^{*}}\right)$ is called the binary closure of $(A, B)$, denoted by $b$-cl $(A, B)$ in the binary space $(X, Y, M)$ where $(A, B) \subseteq(X, Y)$.

Proposition 3.6. Let $(A, B) \subseteq(X, Y)$. Then $(A, B)$ is binary closed in $(X, Y, M)$ if and only if $(A, B)$ $=b-c l(A, B)$.

Proof. Suppose $(A, B)$ is binary closed in $(X, Y, M)$. By using Definition 3.5, $(A, B) \subseteq b$-cl( $A, B)$ that implies $A \subseteq(A, B)^{1^{*}}$ and $B \subseteq(A, B)^{2^{*}}$. Since $(A, B)$ is a binary closed set containing $(A, B)$,

$\left((A, B)^{1^{*}},(A, B)^{2^{*}}\right) \subseteq(A, B)$. This proves that $(A, B)=b-c l(A, B)$

Conversely, let $(A, B)=b-c l(A, B)$. By using Proposition 3.4, b-cl(A, B) is binary closed and hence $(A, B)$ is binary closed.

Proposition 3.7. Suppose $(A, B) \subseteq(C, D) \subseteq(X, Y)$ and $(X, Y, M)$ is a binary space. Then

(i) $\quad b-c l(\varnothing, \varnothing)=(\varnothing, \varnothing)$ and $b-c l(X, Y)=(X, Y)$.

(ii) $\quad(A, B) \subseteq b-c l(A, B)$. 


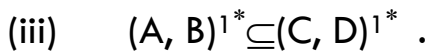

(iv) $\quad(A, B)^{2^{*}} \subseteq(C, D)^{2^{*}}$.

(v) $\quad b-c /(A, B) \subseteq b-c /(C, D)$.

(vi) $\quad b-c l(b-c l(A, B))=b-c l(A, B)$.

Proof. The properties (i) and (ii) follow easily.

Now $(A, B)^{1^{*}}=\cap\left\{A_{\alpha}:\left(A_{\alpha}, B_{\alpha}\right)\right.$ is binary closed and $\left.(A, B) \subseteq\left(A_{\alpha}, B_{\alpha}\right)\right\}$

$$
\begin{aligned}
& \subseteq \cap\left\{A_{\alpha}:\left(A_{\alpha}, B_{\alpha}\right) \text { is binary closed and }(C, D) \subseteq\left(A_{\alpha}, B_{\alpha}\right)\right\} \\
& =(C, D)^{1^{*}} \text {. This proves (iii). }
\end{aligned}
$$

The proof for (iv) is analog. Now, $b-c l(A, B)=\left((A, B)^{1^{*}},(A, B)^{2^{*}}\right) \subseteq\left((C, D)^{1{ }^{*}},(C, D)^{2^{*}}\right)=b-c l(C, D)$ that establishes ( $v$ ). The result (vi) follows from Proposition 3.6.

Theorem 3.8. Let $(A, B)$ and $(C, D)$ be contained in $(X, Y)$ where $(X, Y, M)$ is a binary space. Then

(i) $(A, B)^{1^{*}} \cup(C, D)^{1^{*}} \subseteq(A \cup C, B \cup D) 1^{1 *}$.

(ii) $(A, B)^{2^{*}} \cup(C, D)^{2^{*}} \subseteq(A \cup C, B \cup D) 2^{2^{*}}$.

Proof. Since $(A, B)$ and $(C, D)$ are contained in $(A \cup C, B \cup D)$, by applying Proposition 3.7 (iii)

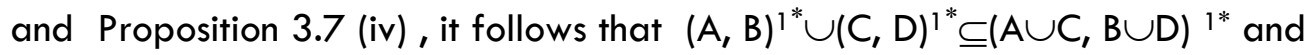
$(A, B)^{2^{*}} \cup(C, D)^{2^{*}} \subseteq(A \cup C, B \cup D) 2^{2^{*}}$. This proves (i) and (ii).

Theorem 3.9. Let $(A, B)$ and $(C, D)$ be contained in $(X, Y)$ where $(X, Y, M)$ is a binary space. Then (i) $(A \cap C, B \cap D){ }^{1 *} \subseteq(A, B)^{1 *} \cap(C, D)^{1 *}$ and

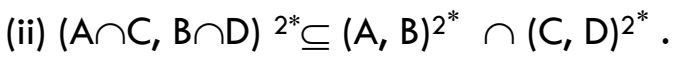

Proof. Analogous to Theorem 3.8.

Remark 3.10. Examples can be constructed to show that the reverse inclusions in Theorem 3.8 and Theorem 3.9 do not hold in general.

Proposition 3.11. Let ( $X, Y, M)$ be a binary topological space and $(A, B) \subseteq(X, Y)$. Let $(A, B))^{\circ}=\cup\left\{A_{\alpha}:\left(A_{\alpha}, B_{\alpha}\right)\right.$ is binary open and $\left.\left(A_{\alpha}, B_{\alpha}\right) \subseteq(A, B)\right\}$ and 
$(A, B)^{2^{\circ}}=\cup\left\{B_{\alpha}:\left(A_{\alpha}, B_{\alpha}\right)\right.$ is binary open and $\left.\left(A_{\alpha}, B_{\alpha}\right) \subseteq(A, B)\right\}$. Then $\left((A, B)^{\circ},(A, B)^{2^{\circ}}\right)$ is binary open and $\left((A, B)^{1^{\circ}},(A, B)^{\circ}\right) \subseteq(A, B)$.

Proof. Follows from Definition 2.1.

The next definition is a consequence of the above proposition .

Definition 3.12. The ordered pair $\left((A, B)^{1^{\circ}},(A, B)^{2^{\circ}}\right)$ is called the binary interior of $(A, B)$, denoted by b-int (A, B).

Proposition 3.13. Let $(A, B) \subseteq(X, Y)$. Then $(A, B)$ is binary open in $(X, Y, M)$ if and only if $(A, B)=$ b- $\operatorname{int}(A, B)$.

Proof. Suppose $(A, B)$ is binary open in $(X, Y, M)$. Since $(A, B)$ is binary open, by Proposition 3.11, $A \subseteq(A, B)^{1^{\circ}}$ and $B \subseteq(A, B)^{\circ}$ that implies $(A, B) \subseteq\left((A, B)^{1^{\circ}},(A, B)^{2^{\circ}}\right)$. Again by using Proposition 3.11, we have $\left((A, B)^{1^{\circ}},(A, B)^{2}\right) \subseteq(A, B)$. This together with Definition 3.12, it follows that $b-\operatorname{int}(A, B)=(A, B)$.

Proposition 3.14. The point $(x, y)$ belongs to $b$ - int $(A, B)$ if and only if there exists a binary neighborhood $(U, V)$ of $(x, y)$ such that $(U, V) \subseteq(A, B)$.

Proof. Suppose there exists a binary neighborhood $(U, V)$ of $(x, y)$ such that $(U, V) \subseteq(A, B)$. Then $U \subseteq(A, B)^{\circ}$ and $V \subseteq(A, B)^{2^{\circ}}$ that implies $(x, y) \in b$-int $(A, B)$. Conversely, let $(x, y) \in b$-int $(A, B)$. Then by using Definition 3.12, there binary open sets $\left(A_{1}, B_{1}\right) \subseteq(A, B)$ and $\left(A_{2}, B_{2}\right) \subseteq$ $(A, B)$ such that $x \in A_{1}$ and $y \in B_{2}$ so that $\left(A_{1} \cup A_{2}, B_{1} \cup B_{2}\right) \subseteq(A, B)$ is a binary neighbourhood of $(x, y)$.

The following propositions that are analogous to Proposition 3.7, Proposition 3.8 and Proposition 3.9 , can be easily established.

Proposition 3.15. Suppose $(A, B) \subseteq(C, D) \subseteq(X, Y)$ and $(X, Y, M)$ is a binary space. Then

(i) $\quad b-\operatorname{int}(\varnothing, \varnothing)=(\varnothing, \varnothing)$ and $b-\operatorname{int}(X, Y)=(X, Y)$.

(ii) $\quad b$-int $(A, B) \subseteq(A, B)$.

(iii) $\quad(A, B)^{1^{\circ}} \subseteq(C, D) 1^{\circ}$.

(iv) $\quad(A, B)^{\circ} \subseteq(C, D)^{2^{\circ}}$.

(v) $\quad b$-int $(A, B) \subseteq b-\operatorname{int}(C, D)$.

(vi) $\quad b$-int $(b-\operatorname{int}(A, B))=b-\operatorname{int}(A, B)$. 
Proposition 3.16. Let $(A, B)$ and $(C, D)$ be contained in $(X, Y)$ where $(X, Y, M)$ is a binary space. Then

$$
\begin{aligned}
& \text { (i) }(A, B) 1^{\circ} \cup(C, D) 1^{\circ} \subseteq(A \cup C, B \cup D) 1^{\circ} . \\
& \text { (ii) }(A, B) 2^{\circ} \cup(C, D) 2^{\circ} \subseteq(A \cup C, B \cup D)^{2^{\circ}} .
\end{aligned}
$$

Proposition 3.17. Let $(A, B)$ and $(C, D)$ be contained in $(X, Y)$ where $(X, Y, M)$ is a binary space. Then

(i) $(A \cap C, B \cap D) 1^{\circ} \subseteq(A, B) 1^{\circ} \cap(C, D) 1^{\circ}$.

(ii) $(A \cap C, B \cap D)^{2} \subseteq(A, B)^{2^{\circ}} \cap(C, D)^{2^{\circ}}$.

Remark 3.18. Examples can be constructed to show that the reverse inclusions in Theorem 3.16 and Theorem 3.17 do not hold in general.

\section{Binary continuity}

Continuity between topological spaces play a dominant role in analysis. In this section the concept of binary continuity between a topological space and a binary topological space is introduced and its basic properties are studied.

Definition 4.1. Let $f: Z \rightarrow X \times Y$ be a function. Let $A \subseteq X$ and $B \subseteq Y$. We define $f^{-1}(A, B)=\{z \in Z: f(z)=(x, y) \in(A, B)\}$.

Definition 4.2. Let $(X, Y, M)$ be a binary topological space and let $(Z, \tau)$ be a topological space. Let $f: Z \rightarrow X \times Y$ be a function. Then $f$ is called binary continuous if $f^{-1}(A, B)$ is open in $Z$ for every binary open set $(A, B)$ in $X \times Y$.

The following lemma will be used in the proof of Proposition 4.5.

Lemma 4.3. Let $f: Z \rightarrow X \times Y$ be a function. For $A \subseteq X$ and $B \subseteq Y$, we have $Z \backslash f^{-1}(A, B)=f^{-1}(A, Y \backslash B) \cup f^{-1}(X \backslash A, B) \cup f^{-1}(X \backslash A, Y \backslash B)$.

Proof. Let $(x, y)=f(z) . z \in f^{-1}(X \backslash A, Y \backslash B) \Rightarrow f(z) \in(X \backslash A, Y \backslash B) \Rightarrow(x, y) \in(X \backslash A, Y \backslash B)$

$$
\begin{gathered}
\Rightarrow x \in X \backslash A \text { and } y \in Y \backslash B \Rightarrow x \notin A \text { and } y \notin B . \\
\Rightarrow(x, y) \notin(A, B) \Rightarrow f(z) \notin(A, B) \Rightarrow z \notin f^{-1}(A, B) \\
\Rightarrow z \in Z \backslash f^{-1}(A, B) .
\end{gathered}
$$

Thus, $f^{-1}(X \backslash A, Y \backslash B) \subseteq Z \backslash f^{-1}(A, B)$. 
$z \in f^{-1}(A, Y \backslash B) \Rightarrow f(z) \in(A, Y \backslash B) \Rightarrow(x, y) \in(A, Y \backslash B)$ where $(x, y)=f(z)$.

$$
\begin{aligned}
& \Rightarrow x \in A \text { and } y \in Y \backslash B \Rightarrow x \in A \text { and } y \notin B \\
& \Rightarrow(x, y) \notin(A, B) \Rightarrow f(z) \notin(A, B) \Rightarrow z \notin f^{-1}(A, B) \Rightarrow z \in Z \backslash f^{-1}(A, B) .
\end{aligned}
$$

Thus, $f^{-1}(A, Y \backslash B) \subseteq Z \backslash f^{-1}(A, B)$.

Similarly we can prove that $f^{-1}(X \backslash A, B) \subseteq Z \backslash f^{-1}(A, B)$

The above arguments show that $f^{-1}(A, Y \backslash B) \cup f^{-1}(X \backslash A, B) \cup f^{-1}(X \backslash A, Y \backslash B) \subseteq Z \backslash f^{-1}(A, B)$.

Now $z \in Z \backslash f^{-1}(A, B) \Rightarrow z \in Z$ and $z \notin f^{-1}(A, B) \Rightarrow z \in Z$ and $f(z) \notin(A, B)$

$$
\Rightarrow z \in Z \text { and }(x, y) \notin(A, B) \text { where } f(z)=(x, y)
$$

$\Rightarrow z \in Z$ and $(x, y) \in(A, Y \backslash B)$ or $(x, y) \in(X \backslash A, B)$ or $(x, y) \in(X \backslash A, Y \backslash B)$

$\Rightarrow z \in Z$ and $f(z) \in(A, Y \backslash B)$ or $f(z) \in(X \backslash A, B)$ or $f(z) \in(X \backslash A, Y \backslash B)$

$\Rightarrow z \in Z$ and $z \in f^{-1}(A, Y \backslash B)$ or $z \in f^{-1}(X \backslash A, B)$ or $z \in f^{-1}(X \backslash A, Y \backslash B)$

$\Rightarrow z \in f^{-1}(A, Y \backslash B) \cup f^{-1}(X \backslash A, B) \cup f^{-1}(X \backslash A, Y \backslash B)$.

Thus $Z \backslash f^{-1}(A, B) \subseteq f^{-1}(A, Y \backslash B) \cup f^{-1}(X \backslash A, B) \cup f^{-1}(X \backslash A, Y \backslash B)$.

Therefore $Z \backslash f^{-1}(A, B)=f^{-1}(A, Y \backslash B) \cup f^{-1}(X \backslash A, B) \cup f^{-1}(X \backslash A, Y \backslash B)$.

Proposition 4.4. Let $(Z, \tau)$ be a topological space and $(X, Y, M)$ be a binary topological space. Let $f: Z \rightarrow X \times Y$ be a function such that $Z \backslash f^{-1}(A, B)=f^{-1}(X \backslash A, Y \backslash B)$ for all $A \subseteq X$ and $B \subseteq Y$. Then $f$ is binary continuous if and only if $f^{-1}(A, B)$ is closed in $Z$ for all binary closed sets $(A, B)$ in $(X$, $Y, M)$.

Proof. Assume that $f$ is binary continuous.

Let $(A, B) \in X \times Y$ be a binary closed set. Therefore, $(X \backslash A, Y \backslash B)$ is binary open set.

That is $(X \backslash A, Y \backslash B) \in M$. Since $f$ is binary continuous, we have $f^{-1}(X \backslash A, Y \backslash B)$ is open in $Z$. Therefore $Z \backslash f^{-1}(A, B)$ is open in $Z$. Hence, $f^{-1}(A, B)$ is closed in $Z$.

Conversely, assume that if $f^{-1}(A, B)$ is closed in $Z$ for all binary closed set $(A, B)$ in ( $\left.X, Y, M\right)$.

Let $(A, B) \in X \times Y$ be a binary open set. To prove $f^{-1}(A B)$ is open $Z$. Since $(A, B) \in M$, we have $(X \backslash A, Y \backslash B)$ is binary closed set in $X \times Y$. Therefore, by our assumption $f^{-1}(X \backslash A, Y \backslash B)$ is closed in Z. Thus, $Z \backslash f^{-1}(A, B)$ is closed in $Z$. Hence $f^{-1}(A, B)$ is open in $Z$. This proves that $f$ is binary continuous. 
Proposition 4.5. Let $(X, Y, M)$ be a binary topological space such that $(A, Y \backslash B)$ and $(X \backslash A, B)$ are binary open in $(X, Y, M)$ whenever $(A, B)$ is binary open. Then $f: Z \rightarrow X \times Y$ is binary continuous if and only if $f^{-1}(A, B)$ is closed in $Z$ for all binary closed set $(A, B)$ in $(X, Y, M)$.

Proof. Assume that $f: Z \rightarrow X \times Y$ is binary continuous. Let $(A, B) \in X \times Y$ be a binary closed set. Therefore, $(X \backslash A, Y \backslash B)$ is a binary open set. That is, $(X \backslash A, Y \backslash B) \in M$. Since $f$ is binary continuous, we have $f^{-1}(X \backslash A, Y \backslash B)$ is open in $Z$. Since $(A, Y \backslash B)$ and $(X \backslash A, B)$ are binary open in $(X, Y, M$ ), by Lemma 4.3 we have $Z \backslash f^{-1}(A, B)$ is open in $Z$. Hence, $f^{-1}(A, B)$ is closed in $Z$.

Conversely, assume that $f^{-1}(A, B)$ is closed in $Z$ for all binary closed sets $(A, B)$ in $(X, Y, M)$. Let $(A, B) \in X \times Y$ be a binary open set. To prove $f^{-1}(A, B)$ is open in $Z$. Since $(A, B) \in M$, we have $(X \backslash A, Y \backslash B)$ is binary closed set in $X \times Y$. Therefore, by our assumption $f^{-1}(X \backslash A, Y \backslash B)$ is closed in Z.

Proposition 4.6. $f: Z \rightarrow X \times Y$ is binary continuous if and only if for every $z \in Z$ and for every binary open set $(A, B)$ with $f(z) \in(A, B)$ there is an open set $U \subseteq Z$ such that $f(U) \subseteq(A, B)$.

Proof. Assume that $f: Z \rightarrow X \times Y$ is binary continuous. Let $(A, B)$ be a binary open set with $f(z)=(x$, $y) \in(A, B)$. Then $z \in f^{-1}(A, B)$. Take $U=f^{-1}(A, B)$. Then $U$ is an open set in $Z$ with $z \in U$. Also $f(U)$ $=\{f(U): U \in U\} \subseteq(A, B)$.

Conversely, we assume that for all $z \in Z$ and for every binary open set $(A, B)$ with $f(z) \in(A, B)$ there exists an open set $U$ in $Z$ with $z \in U, f(U) \subseteq(A, B)$. Let $(A, B)$ be a binary open set. To show that $f^{-1}(A, B)$ is open in $Z$. Let $u \in f^{-1}(A, B)$. Then

$f(U) \in(A, B)$. By our assumption there exists an open set $U$ with $f(U) \subseteq(A, B)$. Therefore, $f^{-1} f(U) \subseteq f^{-1}(A, B)$. That is $U \subseteq f^{-1}(A, B)$. This shows that for each $U \in f^{-1}(A, B)$ there is an open set $U$ containing $U$ such that $U \subseteq f^{-1}(A, B)$ that implies $f^{-1}(A, B)$ is a union of open sets in $Z$. This proves that $f^{-1}(A, B)$ is open in $Z$ that implies $f$ is binary continuous.

Proposition 4.7. $f: Z \rightarrow X \times Y$ is binary continuous if and only if for every $A \subseteq X$ and $B \subseteq Y$, $f^{-1}(b-\operatorname{int}(A, B)) \subseteq \operatorname{int}\left(f^{-1}(A, B)\right)$.

Proof. Suppose $f: Z \rightarrow X \times Y$ is binary continuous. Let $A \subseteq X$ and $B \subseteq Y$. Then by Proposition 3.1 1, $b-$ $\operatorname{int}(A, B)$ is binary open in $(X, Y, M)$ and contained in $(A, B)$. Therefore, $f^{-1}(b$-int $(A, B))$ is open in Z. 
Now, b-int $(A, B) \subseteq(A, B) \Rightarrow f^{-1}(b-\operatorname{int}(A, B)) \subseteq f^{-1}(A, B)$

$$
\begin{aligned}
& \Rightarrow \operatorname{int} f^{-1}(b-\operatorname{int}(A, B)) \subseteq \operatorname{int} f^{-1}(A, B) . \\
& \Rightarrow f^{-1}(b-\operatorname{int}(A, B)) \subseteq \operatorname{int} f^{-1}(A, B) .
\end{aligned}
$$

Conversely, assume that $f^{-1}(b-\operatorname{int}(A, B)) \subseteq \operatorname{int} f^{-1}(A, B)$ for every $A \subseteq X$ and $B \subseteq Y$. Let $(A, B) \in M$. Then $b-\operatorname{int}(A, B)=(A, B)$. Therefore, $f^{-1}(A, B)=f^{-1}(b-\operatorname{int}(A, B)) \subseteq \operatorname{int} f^{-1}(A, B)$. Therefore, intf ${ }^{-1}(A, B)$ is open in $Z$.

\section{Acknowledgments}

The authors are thankful to the referee for the valuable suggestions given to improve the quality of the article.

\section{Reference}

[1] Ryszard Engelking (1977) Generel Topology, Polish Scientific Publishers, Warszawa . 